\title{
Vergleich der Einleitungen in deutschen und in chinesischen Abschlussarbeiten
}

\author{
Shing-Lung Chen (Kaohsiung)
}

\begin{abstract}
Wissenschaftliches Schreiben an der Universität ist für die Studierenden schwierig und führt nicht selten sogar zum Abbruch des Studiums (vgl. Ehlich/Steets 2003; Dittmann/Geneuss/ Nennstiel/Quast 2003; Steinhoff 2007). Bisher wurden Abschlussarbeiten nur zwischen europäischen Sprachen verglichen (vgl. Kaiser 2003), aber nicht zwischen Deutsch und Chinesisch. In diesem Artikel werden Unterschiede in den Makrostrukturen von Abschlussarbeiten aus beiden Ländern analysiert und dazu authentische Belege herangezogen. Dabei werden als Forschungsmethoden sowohl die Move Analysis als auch statistische Analysen verwendet. Als Ergebnis werden deutliche Unterschiede zwischen deutschen und chinesischen Abschlussarbeiten in Bezug auf die Makrostruktur berichtet und deren Bedeutung diskutiert.
\end{abstract}

\section{$1 \quad$ Einleitung}

Sowohl in Deutschland als auch in Taiwan müssen die Studierenden vor ihrem Studienabschluss eine Abschlussarbeit verfassen. Diese dient zum Erlangen eines akademischen Titels. Je nach Studiengang werden solche Arbeiten „Magisterarbeit“, „Diplomarbeit“, „Bachelor-,, oder „Masterarbeit“ genannt. Hier wird einheitlich von „Abschlussarbeit" gesprochen und dieser Beitrag konzentriert sich auf die Magisterarbeiten beider Sprachen. Nach Ehlich/Steets (2003: 1) ist das Wissen um wissenschaftliches Schreiben für den akademischen Diskurs sehr wichtig:

Schreiben ist ein zentrales Medium wissenschaftlicher Kommunikation. Die Fähigkeit, wissenschaftliche Texte zu verfassen, ist eine Kompetenz, die den Erfolg in Wissenschaft und Studium wesentlich mitbestimmt.

(Ehlich/Steets 2003: 1)

Jedoch ist das Verfassen einer wissenschaftlichen Arbeit auch für deutsche muttersprachliche Studierende schwierig. Dazu Steinhoff (2007: 1):

Da ein erfolgreiches Studium maßgeblich von wissenschaftlichen Schreibfähigkeiten abhängt, führen die Probleme vielfach zur Verlängerung der Studienzeiten, nicht selten sogar zum Abbruch des Studiums.

(Steinhoff 2007: 1)

Dittmann/Geneuss/Nennstiel/Quast (2003: 158) und Kruse/Jakobs (1999: 25) vertreten die Ansicht, dass das Verfassen wissenschaftlicher Arbeiten für Muttersprachler schon schwierig, 
für die ausländischen Studierenden noch schwieriger sei. Dies gilt auch für taiwanische Studierende der Germanistik. Der Grund liegt darin, dass es für Ausländer beim deutschen wissenschaftlichen Schreiben nicht nur um die Sprachverwendung geht, sondern auch um die Organisation der Wissensstruktur sowie deutsche wissenschaftliche Konventionen. Da deutsche und chinesischsprachige Magisterarbeiten kaum miteinander verglichen werden, fehlen bisher authentische Belege darüber, welche Divergenzen zwischen beiden bestehen, und somit auch Ratschläge, wie man dem deutschen Verstehensprozess entsprechend Abschlussarbeiten schreiben sollte. Deswegen ist es notwendig, deutsche Magisterarbeiten für taiwanische Studierende zu analysieren.

Nach Gruber/Huemer (2008: 332) werden in deutschsprachigen Ländern vor allem Fachartikel analysiert, aber kaum die Arbeiten von Studierenden. Auch Hsiao/Yu (2012: 133), die englischsprachige Magisterarbeiten von taiwanischen Studierenden untersuchen, bemerken, dass wissenschaftliche Arbeiten von Studierenden kaum analysiert werden. Steinhoff (2007: 2) weist auf die Wichtigkeit des wissenschaftlichen Schreibens hin und meint, dass es nicht nur für das Studium, sondern für das ganze Leben als eine zentrale Kompetenz betrachtet wird.

Nach Şenöz-Ayata (2012: 35-36) und Huemer (2014: 122) wird je nach Sprache (Kultur) und Fachdisziplin ein wissenschaftlicher Text anders organisiert. Loi/Evans (2010: 2820) analysieren die Unterschiede der rhetorischen Organisation zwischen englischen und chinesischen Forschungsartikeln und kommen zum Ergebnis, dass chinesische und englische Leser andere Erwartungen an wissenschaftliche Texte stellen.

EAP writing instructors should be aware that Chinese students might base their EAP writing on a set of rhetorical forms that differ from those of English. This should allow them to make informed pedagogical decisions that are grounded in the understanding of the preferred rhetorical strategies in both languages, to guide Chinese students in writing English academic writings which are acceptable and comprehensible to English audiences.

In den Untersuchungen über das wissenschaftliche Schreiben von Studierenden wurden meistens Hausarbeiten von Muttersprachlern analysiert, aber kaum die der Nichtmuttersprachler. In Österreich untersuchten Gruber et al. (2006) die deutschen Proseminar- und Seminararbeiten österreichischer Studierenden. In Deutschland verglich Steinhoff (2007: 3) die Hausarbeiten der Studierenden mit Fachzeitschriftenartikeln von geisteswissenschaftlichen Wissenschaftlern (siehe dazu Kapitel 2).

Ehlich/Steets (2003) und Redder (2002) beschäftigten sich mit dem wissenschaftlichen Schreiben von Nichtmuttersprachlern. Aber sie analysieren die wissenschaftlichen Arbeiten ausländischer Studierenden in der Regel, ohne diese jedoch nach Herkunftskultur zu differenzieren (vgl. Kapitel 2). Ebenfalls fehlt ein kontrastiver Vergleich wissenschaftlicher Texte zwischen deutschen und chinesischen Studierenden.

Da entsprechende Forschungsergebnisse fehlen, ist es in Taiwan schwierig, den Studierenden konkrete Ratschläge und authentische Belege $\mathrm{zu}$ deutschen wissenschaftlichen Arbeiten vorzulegen. Deswegen bereitet das Schreiben einer Magisterarbeit den taiwanesischen Studierenden viel Mühe und Schwierigkeiten. Dieser Artikel vergleicht die Makrostruktur deutschsprachiger Magisterarbeiten deutscher und österreichischer Studierender mit 
derjenigen von chinesischsprachigen Magisterarbeiten taiwanischer Studierender, um deren Unterschiede zu erkunden und Belege für wissenschaftliches Schreiben zu ermitteln. Damit soll es möglich werden, den taiwanischen Studierenden beim Schreiben von Magisterarbeiten Hilfestellungen anzubieten (siehe Kapitel 3: Forschungsmaterial und Kapitel 4: Empirische Analyse und Ergebnisse) $)^{1}$.

Da jeder Textteil in Fachartikeln wie z. B. „Einleitung“, „Forschungsstand“, „Schlussteil“ eine andere Makrostruktur aufweist, behandelt die Forschung meistens nur einen Textteil. Auch hier wird nur auf den Einleitungsteil von Abschlussarbeiten Bezug genommen².

Die Fragestellungen der vorliegenden Untersuchung sind:

(1) Wie werden die Einleitungen in deutschen und in chinesischen Magisterarbeitenorganisiert?

(2) Worin bestehen die Unterschiede und Gemeinsamkeiten zwischen den beiden?

Im Folgenden geht dieser Beitrag zunächst auf den Forschungsstand ein und stellt dar, inwiefern wissenschaftliche Arbeiten von Studierenden Gegenstand der Forschung sind. Daraufhin wird dargelegt, welche Abschlussarbeiten als Forschungsmaterial sich für einen Vergleich eignen und worin die Vergleichsbasis zwischen deutschen und chinesischen Magisterarbeiten besteht. Anschließend wird erläutert, mit welchen Forschungsmethoden das Untersuchungsmaterial analysiert wird. Zum Schluss wird auf die ermittelten Unterschiede zwischen deutschen und chinesischen Magisterarbeiten eingegangen.

\section{Forschungsstand}

Die bisherige Forschung über wissenschaftliches Schreiben lässt sich in zwei Hauptrichtungen einteilen:

(1) Analyse von Fachartikeln von Forschenden und Wissenschaftlern

(2) Analyse von wissenschaftlichen Arbeiten von Studierenden (wie z. B. Seminararbeit, Magisterarbeit etc.).

Es liegt eine Reihe von Untersuchungen über Fachtexte vor (vgl. Clyne 1987; Sachtleber 1990; Graefen 1997; Thielmann 2009; Şenöz-Ayata 2012; Tzilinis 2013 etc.), während relativ wenig Literatur über das wissenschaftliche Schreiben ausländischer Studierenden in deutschsprachigen Kontexten zu finden ist (vgl. Redder 2002; Ehlich/Steets 2003; Gruber et al. 2006; Pohl 2007; Steinhoff 2007).

Die Forschung über das wissenschaftliche Schreiben lenkte ihre Aufmerksamkeit zunächst auf die interkulturellen Unterschiede. Jeder Kulturkreis hat seinen eigenen Verstehensprozess,

\footnotetext{
1 Vor dem 2. Weltkrieg gehörten China und Taiwan demselben Staat und haben dieselbe Amtssprache „Mandarin-Chinesisch“. Da „Taiwanisch“ ein Dialekt ist und in Taiwan beim wissenschaftlichen Schreiben Chinesisch verwendet wird, gibt es keine taiwanischen Fachartikel, wohl aber chinesische Fachartikel aus Taiwan.

${ }^{2}$ Der Textteil wird „Einleitung“ genannt, jedoch meistens erst verfasst, nachdem der Hauptinhalt des Artikels bestimmt worden ist. Dazu Swales (2003: 173): "It is widely recognized that writing introductions is slow, difficult, and troublesome for both native speakers as well as nonnative speakers".
} 
was in der wissenschaftlichen Textproduktion oft $\mathrm{zu}$ eigenen und sehr spezifischen Makrostrukturen führt. Gruber (2010: 17-18) weist darauf hin, dass in den USA Untersuchungen vorliegen, die zeigen, warum bei Studierenden aus verschiedenen Ländern unterschiedliche Probleme im wissenschaftlichen Schreiben vorkommen. Sowohl ŞenözAyata (2012: 35-36) als auch Huemer (2014: 122) kommen zum Ergebnis, dass Fachartikel je nach Kultur und Fachdisziplin anders organisiert sind. Dittmann/Geneuss/Nennstiel/ Quast 2003 vertreten auch die ähnliche Ansicht:

Wissenschaftliche Texte weisen spezifische Merkmale auf, die in Abhängigkeit vom Fach (z. B. geisteswissenschaftliche vs. naturwissenschaftliche Texte; vgl. Jakobs 1999, 174ff), von kulturspezifischen Faktoren (z. B. deutsches „Referat“"vs. US-amerikanischer „Essay“) und von der jeweiligen spezifischen Gattung (z. B. Forschungsüberblick vs. Empirischer Beitrag) variieren.

(ebd.: 158)

Daran kann man erkennen, dass die Besonderheiten des wissenschaftlichen Schreibens sowohl mit der Sprache (Kultur) als auch mit der Fachdisziplin zusammenhängen. Daraus ist es anzunehmen, dass dieselbe Textsorte wie z. B. „Magisterarbeit“ in verschiedenen Sprachen (Kulturen) eigene Besonderheiten aufweist. Deswegen kann man die Ergebnisse des Vergleiches zwischen englischen und chinesischen wissenschaftlichen Arbeiten nicht auf andere Sprachenpaare übertragen. Zudem muss die Analyse auf wissenschaftliche Arbeiten aus derselben Fachdisziplin beschränkt sein. Somit konzentriere ich mich im Folgenden auf die Analysen wissenschaftlicher Texte von Studierenden aus dem Fachbereich „Germanistische Linguistik“. Es lassen sich zwei Analyserichtungen festmachen:

(a) Analyse wissenschaftlicher Texte von Studierenden mit Deutsch als L1;

(b) Analyse wissenschaftlicher Texte von Studierenden, deren L1 nicht Deutsch ist.

\subsection{Analyse wissenschaftlicher Texte von Studierenden mit Deutsch als L1}

Gruber et al. (2006) untersuchen die Proseminar- und Seminararbeiten von Muttersprachlern aus drei sozialwissenschaftlichen Fächern (Sozial- und Wirtschaftsgeschichte, Personalwirtschaft und Wirtschaftspsychologie). Dabei analysieren sie deren Makro- und Mikrostruktur. Neben der Textanalyse vergleichen sie die Seminararbeiten mit den Anforderungen des Instituts an diese, um herauszufinden, welche sprachlichen Merkmale einer Seminararbeit mit ihrer Beurteilung durch die Seminarleitenden korrespondieren.

In Deutschland untersucht Steinhoff (2007: 3) insgesamt 296 Hausarbeiten von 72 Studierenden und 99 Fachzeitschriftenartikel von Wissenschaftlern aus den Geisteswissenschaften und vergleicht diese miteinander. Außerdem untersucht er die Hausarbeiten der Studierenden während verschiedener Studienphasen, um den Entfaltungsprozess deren wissenschaftlicher Textkompetenz zu ermitteln (ebd.: 422). Die ermittelten Ergebnisse zeigen, dass die Studierenden am Anfang des Studiums Probleme mit wissenschaftlichem Schreiben haben, sich im Laufe der Zeit jedoch von der Verwendung der allgemeinen zu einer Verwendung der spezifischen Wissenschaftssprache entwickeln. Dazu Steinhoff: 
Bei der Produktion ihrer ersten Hausarbeiten stehen sie vor komplexen Schreibproblemen, für deren Lösung sie ihre voruniversitär erworbenen kognitiven, kommunikativen und sprachlichen Fähigkeiten ausbauen und spezifizieren müssen.

(ebd.: 419)

Im Laufe der Zeit eignen sich die Studierenden die Wissenschaftssprache an:

Im Bereich der Verfasserreferenz fiel beispielsweise auf, dass die Pronomina „ich“ und „wir“ im Studienverlauf zunehmend seltener, „werden“-Passiv-Modalverb-Konstruktionen dagegen zunehmend häufiger verwendet werden...

(ebd.: 422)

Szurawitzki (2013) ließ Studierende Fachartikel analysieren, kommentieren und dazu Hauptseminarbeiten schreiben. Sein Untersuchungsmaterial bestand aus 18 Seminararbeiten (ebd.: 348). Dabei untersuchte er, ob und inwiefern die Schreibenden „,wertende sprachliche Mittel verwenden“ (ebd.: 354). Der Analyseschwerpunkt dieser Arbeit lag auf „evaluierende[n] Conclusions“ (ebd.: 350). Dabei wurden das sprachliche Mittel „Adjektiv + NP“ am häufigsten verwendet, gefolgt von „Adjektiv“ und „Adverb + Adjektiv“ (ebd.: 354).

\subsection{Analyse wissenschaftlicher Texte von Studierenden, deren L1 nicht Deutsch ist}

In Deutschland haben sowohl Redder (2002) als auch Ehlich/Steets (2003) je einen Sammelband zum Schwerpunkt Wissenschaftliches Schreiben im Studium publiziert. Dabei beschäftigten sich sowohl Ehlich als auch Redder damit, wie man ausländische Studierenden lehrte bzw. wie ausländische Studierende lernten, wissenschaftlich zu schreiben. Im Mittelpunkt standen hauptsächlich die Textarten, die im Studium überwiegen, wie Seminararbeiten (Fischer/Moll 2002: 137) oder Protokolle von Lehrveranstaltungen (Moll 2002: 85-86), aber kaum die Abschlussarbeiten.

Im Sammelband von Redder (2002) wurden wissenschaftliche Texte von Studierenden analysiert, ohne sie nach dem Kulturkreis zu differenzieren. Dagegen ist im Sammelband von Ehlich/Steets (2003) ein Beitrag über den Vergleich wissenschaftlicher Texte von Studierenden aus verschiedenen Ländern aufgenommen, auf den im Folgenden genauer eingegangen werden soll:

Kaiser (2003) untersuchte zunächst die Schreibanleitungen zu wissenschaftlichen Texten und vergleicht dann in einem weiteren Schritt Seminararbeiten aus Venezuela mit solchen aus Deutschland. Sie analysierte die formalen Strukturen in wissenschaftlichen Texten der Studierenden aus den beiden Ländern. Nach Kaiser (ebd.: 309) bestehen die Ergebnisse dieser Untersuchung hauptsächlich darin, dass wissenschaftliche Texte deutscher Studierender die Literaturangaben sowohl im größeren Umfang als auch präziser behandeln als die aus Venezuela. Dazu Kaiser:

Die Untersuchung der studentischen Texte aus Venezuela und Deutschland ergibt deutliche Unterschiede im Umgang mit externen Wissensquellen, sowohl in der Art der Quellenangabe als auch im Umfang der Bibliografie, in der Anzahl der Zitate aus der Primär- oder Sekundärliteratur sowie in der Anzahl objektsprachlicher Beispiele in der Sprachwissenschaft.

(ebd.: 321)

Aus der Analyse von Kaiser lässt sich folgern, dass die Textproduktion von Studierenden in Abhängigkeit ihres Kulturkreises gestaltet wird. Man kann die Ergebnisse von Swales, von $\mathrm{Hsiao} / \mathrm{Yu}$ etc., die ausschließlich wissenschaftliche Textproduktion von englischen 
Studierenden untersucht haben, nicht auf die deutschen Studierenden übertragen. Außerdem wurden in Deutschland bis jetzt kaum Abschlussarbeiten vor Ort untersucht, und diese kaum mit denen anderer Länder verglichen. Ein Vergleich zwischen deutschen und chinesischen Abschlussarbeiten steht bis dato noch aus.

\section{Forschungsmaterial und Forschungsmethoden}

\subsection{Forschungsmaterial}

In dieser Forschung werden zwei Textkorpora analysiert:

(1) Ein Textkorpus chinesischer Magisterarbeiten von taiwanischen Studierenden der Germanistik (30 Magisterarbeiten) und

(2) Ein Textkorpus deutscher Magisterarbeiten von deutschen und österreichischen Studierenden der Germanistik (30 Magisterarbeiten). Damit nicht einseitig nur die Magisterarbeiten aus Deutschland untersucht werden, werden jeweils die Hälfte der Magisterarbeiten aus Deutschland und die andere Hälfte aus Österreich im Korpus aufgenommen.

Um eine Vergleichsbasis zwischen den Magisterarbeiten beider Sprachen zu schaffen, werden nur die Arbeiten, die zum Erlangen des akademischen Titels Magister bzw. Master akzeptiert wurden, als Untersuchungsmaterial aufgenommen. Alle Arbeiten stammen aus dem Zeitraum vom Jahr 2000 bis 2013.

Mit den deutschsprachigen Abschlussarbeiten aus Deutschland und aus Österreich werden die auf Chinesisch geschriebenen Arbeiten aus Taiwan verglichen, aber nicht die auf Deutsch geschriebenen aus Taiwan. Die Gründe ergeben sich wie folgt:

(1) Die auf Chinesisch geschriebenen Magisterarbeiten taiwanischer Studierender stellen die ursprünglichen Schreibkonventionen der Taiwaner dar.

(2) Dieser Artikel analysiert keine Sprachverwendung in beiden Sprachen, sondern das Schreibmodell (also die Makrostruktur) in den jeweiligen Muttersprachen.

Die Vergleichsbasis der Magisterarbeiten beider Länder besteht darin, dass sie beide aus dem Fach „Germanistischer Linguistik“ stammen. Außerdem bestehen sie in derselben Textsorte und beruhen auf einem vergleichbaren Wissensniveau in Bezug auf Studien- und Textkompetenz. Durch den kontrastiven Vergleich zwischen deutschen und chinesischen Abschlussarbeiten lassen sich Ergebnisse erzielen, aus denen die Studierenden aus Taiwan die spezifisch chinesischen Textsortenkonventionen erkennen und daraus dann die deutschen Textsortenkonventionen erschließen können.

\subsection{Forschungsmethoden}

Für die Analyse der Makrostrukturen und die Ermittlung der Verwendungshäufigkeit bestimmter Struktur in Magisterarbeiten werden hier statistische Methoden und die aus dem CARS-Modell entstandene Move Analysis verwendet. Im Folgenden wird hauptsächlich dargestellt, wie diese Forschungsmethoden zur Analyse des Forschungsmaterials verwendet werden: 


\subsubsection{Das CARS-Modell (Move Analysis)}

Das CARS-Modell von Swales wird seit 1981 verwendet. Dessen Validität wurde wiederholt unter Beweis gestellt. Li verwendet auch dieses Modell zur Analyse der wissenschaftlichen Fachtexte und begründet es wie folgt:

Das CARS-Modell wurde mehrmals als valid bewiesen. Deswegen wird hier auch das CARSModell verwendet, um den Textteil „Einleitung“ in englischen und in chinesischen sozialwissenschaftlichen Fachartikeln zu vergleichen [Übersetzung; C. S-L.].

(Li 2012: 110)

Nach Swales (1990) wird die Struktur von Einleitungen in Fachartikeln durch das CARSModell dargestellt. Später analysiert er die Einleitungen wissenschaftlicher Arbeiten der Studierenden auch gemäß demselben CARS-Modell (2003: 173-194) und stellt damit die Anwendbarkeit des CARS-Modells auch für wissenschaftliche Arbeiten von Studierenden unter Beweis. Das allgemeine Analyseverfahren wird heute auch Move Analysis genannt (siehe Biber-/Connor/Upton 2007). Dieser Beitrag folgt der Vorgehensweise einer Move Analysis, um die Makrostruktur der Einleitungen in Abschlussarbeiten beider Länder danach zu analysieren, ob sie dem CARS-Modell entsprechen. Das Modell besteht aus den drei in Tabelle 1 dargestellten Moves. Jedem Move werden mehrere Steps zugeordnet. Im Folgenden werden drei Moves mit deren Steps für Einleitungen dargestellt (siehe Tabelle 1) und noch angemerkt, in welcher Literatur Swales die entsprechende Theorie behandelt hat.

Der Textteil „Einleitung“ kann die folgenden Strukturelemente haben. Hier werden mögliche Moves und Steps zusammengestellt, damit ein relativ vollständigerer Überblick angeboten wird:

Move 1 establishing a territory (Feak/Swales 2011; Swales 1990)

Step 1 showing that the general research area is important, central, interesting, problematic, or relevant in some way (optional) (Feak/ Swales 2011)

Step 2 introducing and reviewing items of previous research in the area (obligatory) (Feak/Swales 2011)

Move 2 establishing a niche

Step 1A indicating a gap (Swales1990; Swales 2004; Feak/ Swales 2011)

Step 1B adding to what is known (Swales 2004; Feak /Swales 2011)

Step 2 presenting positive justification (Swales 2004)

Move 3 occupying the niche (Swales 1990; Feak/Swales 2011)

Step 1 announcing present research descriptively and/or purposively (Swales 1990; Swales 2004; Feak /Swales 2011)

Step 2 presenting research questions or hypotheses (Swales 2004)

Step 3 definitional clarifications (Swales 2004)

Step 4 summarizing methods (Swales 2004)

Step 5 announcing principal outcomes (Swales 1990, 2004, 2011)

Step 6 stating the value of the present research (Swales 2004, 2011)

Step 7 outlining the structure of the paper (Swales 1990, 2004, 2011)

Tabelle 1: Elemente (Moves) für Einleitungen wissenschaftlicher Artikel nach Swales und Feak und Swales

In diesem Artikel werden in Anlehnung an das CARS-Modell von Swales die Einleitungen beider Länder danach überprüft, wie weit sie der Makrostruktur nach Swales/Feak und Swales (Tabelle 1) entsprechen. Dieses Vorgehen erlaubt eine qualitative Vergleichbarkeit beider Korpora. Nach Zaleska (2010: 153) ist die Genre-Analyse nach Swales geeignet für den Vergleich zwischen zwei Sprachen, weil wissenschaftliche Artikel der beiden Sprachen nach den gleichen Kriterien klassifiziert werden. 


\subsubsection{Statistische Analysen}

In der vorliegenden Arbeit wird nicht nur die qualitative Methode (also die Move Analysis) verwendet, sondern es kommt auch eine statistisch-quantitative Methode zur Anwendung. Im Folgenden wird hauptsächlich dargestellt, wie die Statistik hier verwendet wird: Die Notwendigkeit der quantitativen Methode ist darin begründet, dass sie das Forschungsmaterial zwar tiefer analysiert, jedoch nichts darüber aussagen kann, ob das analysierte Phänomen mehrheitlich zutrifft. Nach Hussy/Schreier/Echterhoff (2010: 50) stellen quantitative Daten in Form von Zahlen konkrete Informationen dar und erlauben eine vergleichsweise hohe Präzision (ebd.: 51).

Die Vorteile einer statistischen Auswertung bestehen darin, die Reliabilität und Validität der Ergebnisse sicherzustellen (Moore 2002: 330-347). Um die quantitativen Ergebnisse abzusichern, werden hier sowohl der T-Test als auch der Fisher's exact Test verwendet, um genauer Aussagen darüber zu treffen, ob zwischen zwei Mittelwerten ein signifikanter Unterschied besteht sowie ob die ermittelten Ergebnisse zuverlässig und kein Zufall sind (vgl. Bharadwaj 2000: 178).

Die Ergebnisse, die den T-Test bestehen, werden weiterhin mittels des Fisher's exact Testes überprüft, ob bezüglich eines bestimmten Phänomens die Propositionen der zwei zu untersuchenden Daten identisch sind. Zum Grundmodell des Fisher exact Testes erklärt Fisher:

$\chi^{2}$ in fact was the square of the distance of a random point from the centre of a homogeneous normal distribution in $\mathrm{n}$ dimensions. The number of dimensions, however, would be reduced by unity for every restriction upon deviations between expectation and observation.

(Fischer 1922: 336)

Mit diesem Testverfahren werden die Ergebnisse des T-Tests noch einmal verifiziert und erhalten somit eine größere Zuverlässigkeit.

\section{$4 \quad$ Empirische Analyse und Ergebnisse}

Im Folgenden analysieren wir mittels der Move Analysis und der Statistik die Abschlussarbeiten von Studierenden beider Länder. Die Fragestellungen sind wie folgt:

(a) Welche Makrostruktur weisen deutsche und chinesische Einleitungen auf? Worin bestehen die Unterschiede zwischen deutschen und chinesischen Einleitungen?

(b) Welche von den in Tabelle 1 aufgelisteten Moves werden verwendet? Gibt es konstistente Muster? (vgl. Kapitel 3.2.1 und siehe Tabelle 1)?

Nach Swales (2003) sollen Einleitungen in einer wissenschaftlichen Arbeit von Studierenden aus drei Moves bestehen. Falls ein Move fehlt, kann es dazu führen, dass diese Einleitung inhaltlich unklar ist und der Verstehensprozess der Lesenden blockiert wird.

\subsection{Strukturen der Einleitungen in beiden Sprachen}

Die Einleitungen in den untersuchten Abschlussarbeiten werden unterschiedlich nummeriert: entweder als das nullte Kapitel, als das erste oder auch als das zweite Kapitel. Außerdem sind sie von unterschiedlichem Umfang. Während deutsche Einleitungen durchschnittlich 777.6 
$\pm 105,6$ Wörter besitzen, bestehen chinesische Einleitungen durchschnittlich aus 4056,43 $\pm 245,52$ chinesischen Zeichen. Rechnet man chinesische Zeichen in Wörter um und ein chinesisches Zeichen gleich 0.52 deutsches Wort ist, beträgt eine chinesische Einleitung ca. 2109,34 Wörter. Somit sind chinesische Einleitungen durchschnittlich 2,71fach länger als deutsche $^{3}$. Daraus ergibt sich die Frage, wie sie aufgebaut sind ${ }^{4}$. Einzelheiten siehe Tabelle 2 und 3.

\begin{tabular}{|l|l|l|l|}
\hline & $\begin{array}{l}\text { Einleitungen als } 0 \\
\text { Kapitel }\end{array}$ & $\begin{array}{l}\text { Einleitungen als 1. } \\
\text { Kapitel }\end{array}$ & $\begin{array}{l}\text { Einleitungen als } 2 . \\
\text { Kapitel }\end{array}$ \\
\hline DT & $16,67 \%(5)$ & $80,00 \%(24)$ & $3,33 \%(1)$ \\
\hline CH & $0 \%$ & $100 \%(30)$ & $0,00 \%(0)$ \\
\hline Fisher's exact Test & $\mathrm{p}=0,02372070$ & \\
\hline
\end{tabular}

\section{Tabelle 2: Stellung der Einleitungen in den Magisterarbeiten beider Länder DT: deutschsprachige Magisterarbeiten, $\mathrm{CH}$ : chinesischsprachige Magisterarbeiten}

Die Einleitungen werden in deutschen Magisterarbeiten am häufigsten als das 1. Kapitel angesehen (80,00\%) und nur mit 16,67\% als nulltes Kapitel nummeriert. Dagegen kommen die chinesischen Einleitungen nur als das 1. Kapitel (100\%) vor ${ }^{5}$.

In diesem Artikel werden sowohl der T-Test als auch der Fisher's exact Test verwendet, um die statistischen Ergebnisse zu bestätigen. Um Wiederholung zu vermeiden, gehe ich bei der Analyse i. d. R. nur auf die Ergebnisse des Fisher's exact Testes ein. Da der P-Wert vom Fisher's exact Test $(p=0,02372070)$ in diesem Fall größer ist als der Standwert des Signifikanzniveaus $\mathrm{p}=0,001$, besteht kein überzufälliger Unterschied zwischen den analysierten Daten, offensichtlich weil die Mehrheit der Einleitungen in beiden Sprachen als erstes Kapitel gezählt werden (siehe Tabelle 2).

Bezüglich der inhaltlichen Strukturen lassen sie sich die Einleitungen in zwei Gruppen einteilen:

(1) Einleitungen als ein Kapitel ohne Unterteilung in Unterkapitel

(2) Einleitungen mit Unterteilung in verschiedene Unterkapitel, wie die folgenden zwei Beispiele aufzeigen:

(Beleg 1) In deutschsprachiger Arbeit:

1. Einleitung

1.1 Einleitende Worte

1.2 Forschungsfrage und Hypothese

1.3 Aufbau der Arbeit

\footnotetext{
${ }^{3}$ Ein chinesisches Wort besteht aus chinesischen Zeichen. Ein chinesisches Zeichen entspricht 0,52 deutschen Wörtern. Laut dem „Babel Chinesisch-Englisch Parallel Corpus“ ist ein chinesisches Zeichen gleich wie 0,6 englische Wörter (Xiao 2008: 438), und laut dem Parallelkorpus „,de-news“ entspricht ein englisches Wort 0,87 deutschen Wörtern (Koehn 2000). Daher ist ein chinesisches Zeichen gleich 0,52 deutschen Wörtern.

${ }^{4}$ In chinesischsprachigen Magisterarbeiten gibt es vor dem Textteil „Einleitung“ ein Abstract, das kaum in deutschsprachigen Magisterarbeiten zu finden ist.

${ }^{5}$ Ist die Einleitung nicht als Hauptinhalt der jeweiligen Magisterarbeit zu betrachten, wird sie meistens mit einer Null nummeriert. Im Fall, dass sie als Textteil behandelt wird, wird sie entweder als das erste oder als das zweite Kapitel bezeichnet. Erscheint die jeweilige Einleitung als das 2. Kapitel, wird das 1. Kapitel oft als Vorwort bezeichnet.
} 
(Faulhaber 2013: 1)

(Beleg 2) In chinesischsprachiger Arbeit ${ }^{6}$ :

Das erste Kapitel: Einleitung

Das erste Unterkapitel: Motivation und Zweck der Untersuchung

Das zweite Unterkapitel: Forschungsmethoden [Übersetzung; C. S-L.].

(Huang 2003: 1)

Eine Einleitung mit Unterteilung in Unterkapitel verfolgt das Ziel, den Hauptinhalt übersichtlich zu gestalten.

\begin{tabular}{|l|l|l|}
\hline & Einleitung ohne Unterteilung & Einleitung mit Unterteilung \\
\hline DT & $73,33 \%(22)$ & $26,67 \%(8)$ \\
\hline CH & $23,33 \%(7)$ & $76,67 \%(23)$ \\
\hline Fisher's exact Test & $\mathrm{p}=0,0002$ & \multicolumn{2}{|l|}{} \\
\hline
\end{tabular}

Tabelle 3: Struktur der Einleitungen in beiden Sprachen

In deutschen Magisterarbeiten ist eine „Einleitung ohne Unterteilung“ der Regelfall. Dagegen sind „Einleitungen mit Unterteilung“ in chinesischsprachigen Magisterarbeiten sehr beliebt (76,67\%). Der P-Wert ( $\mathrm{p}=0,0002)$ weist den Unterschied als signifikant aus.

Da in chinesischsprachigen Magisterarbeiten Einleitungen mit Unterkapiteln am häufigsten vorkommen, wird hier nur auf die chinesischen Magisterarbeiten eingegangen (vgl. Tabelle 3). Untersucht man weiterhin, welche Unterkapitel in chinesischen Einleitungen vorkommen, erkennt man deutlich, welche inhaltlichen Schwerpunkte dort den chinesischen L1-Sprechenden am wichtigsten ist:

\begin{tabular}{|l|l|l|l|ll|}
\hline CH & Motivation & $\begin{array}{l}\text { Zweck (bzw. } \\
\text { Zielsetzung) }\end{array}$ & Methode & Aufbau \\
\hline & $22,92 \%(22)$ & $19,79 \%(19)$ & $20,83 \%(20)$ & $14,58 \%(14)$ & des \\
\hline CH & Fragestellungen & Hypothesen & $\begin{array}{l}\text { Arbeitsschritte der } \\
\text { Forschung }\end{array}$ & $\begin{array}{l}\text { Eingrenzung } \\
\text { Forschungsumfangs }\end{array}$ \\
\hline & $8,33 \%(8)$ & $2,08 \%(2)$ & $3,12 \%(3)$ & $8,33 \%(8)$ & \\
\hline
\end{tabular}

Tabelle 4: Inhaltliche Schwerpunkte in chinesischen Einleitungen

Für chinesische Einleitungen sind die Motivation (22,92\%), die Methode (20,83\%) und der Zweck der Forschung (19,79\%) am wichtigsten ${ }^{7}$. Betrachtet man die Kategorien „Motivation“ und „Zweck“ $(22,92 \%+19,79 \%=42,71 \%)$ zusammen, die beide auf die Wichtigkeit der jeweiligen Magisterarbeit eingehen, macht dies fast die Hälfte der Fälle aus. Daraus lässt sich schließen, dass die Hauptaufgabe der Einleitungen in chinesischen Magisterarbeiten darin besteht, diese positiv zu präsentieren. Am zweithäufigsten folgen die Unterabschnitte zum „Aufbau der Arbeit“ (14,58\%) und zur „Eingrenzung des

\footnotetext{
${ }^{6}$ In chinesischen Magisterarbeiten aus Taiwan kommt zu deren chinesischem Titel entweder ein entsprechender deutscher oder englischer Titel hinzu. Diese Arbeit übernimmt den Originaltitel des jeweiligen Verfassers. Deswegen erscheint er manchmal in Englisch und in den anderen wieder in Deutsch (siehe Verzeichnis der im Korpus verwendeten Texte).

${ }^{7}$ Je mehr Objekte im Prozentsatz beteiligt werden, desto weniger signifikant wird der Unterschied sein. Der Grund liegt daran, dass der Prozentsatz von verschiedenen Objekten verteilt wird und somit jedes Objekt einen niedrigen Prozentsatz belegt. Da die Unterkapitel unterschiedlich genannt werden, werden hier die am häufigsten vorkommenden behandelt, um die Unterschiede zwischen den Daten zu erkennen.
} 
Forschungsumfangs“ (8,33\%) sowie Unterkapitel zu den „Fragestellungen“ (8,33\%). Bei der Eingrenzung des Forschungsumfangs wird erklärt, aus welchen Gründen oder aufgrund welcher Bedingungen der Umfang der jeweiligen Magisterarbeit beschränkt wird:

(Beleg 3) Eingrenzung des Forschungsumfangs:

Bei solcher Untersuchung wird der Umfang dieser Arbeit auf die deutschen Lehrwerke „Themen aktuell“" und „Lagune“ der Grundstufe und besonders deren Band I und Band II und deren Arbeitsbücher beschränkt [Übersetzung; C. S-L.].

(Chin 2010: 2)

Ein weiteres Beispiel, siehe Huang (2004: 3).

Dagegen spielen für chinesischsprachige Einleitungen „die Darstellung der Hypothesen“ (2,08\%) und „die Angabe der Arbeitsschritte der Forschung“ (3,12\%) fast keine Rolle. Den Arbeitsschritten wird sehr selten ein selbständiges Unterkapitel gewidmet. Das kann daran liegen, dass diese oft zusammen mit dem Aufbau der Arbeit dargestellt werden.

\subsection{Drei Moves in beiden Sprachen}

In dieser Arbeit werden deutsche mit chinesischen Einleitungen verglichen, ohne zwischen Einleitungen ohne Unterteilung und solchen mit Unterteilung $\mathrm{zu}$ differenzieren. Dies geschieht aus den folgenden Gründen:

(1) Die Einleitungen mit Unterteilung in verschiedene Unterkapitel sind im Prinzip länger als die Einleitungen ohne Unterteilung. Trotzes des langen Umfanges behandeln manche Einleitungen mit Unterteilungen doch keinen Move 2 (vgl. die Magisterarbeiten von Lin 2005, Tsai 2009, Chen 2012 etc.). Die Einzelheiten siehe Kapitel 4.2.1. Außerdem untersucht dieser Artikel keine Länge der Einleitungen, sondern deren inhaltlichen Organisationstruktur. Deswegen werden hier sowohl die Einleitungen mit als auch ohne Unterteilungen zusammen analysiert.

(2) Wie in Tabelle 3 dargestellt wird, sind die meisten chinesischen Einleitungen mit Unterteilung und die deutschen Einleitungen dagegen ohne Unterteilungen. Wenn man aber entweder nur die einfachen Einleitungen oder nur die Einleitungen mit Unterteilung miteinander vergleicht, liegt kein ausreichendes Forschungsmaterial aus beiden Sprachen vor. Deswegen analysieren wir die Gesamtmenge der deutschen mit den chinesischen Einleitungen, ohne sie vorher zu differenzieren.

Die Magisterarbeiten beider Sprachen haben sowohl den Move 1 als auch den Move 3 behandelt. Nur einige chinesischen Magisterarbeiten behandeln den Move 2. Um diesen wichtigen Unterschied genauer in den Blick zu nehmen, betrachten wir im Folgenden Move 2 zuerst und dann erst danach Move 1 und Move 3.

\subsubsection{Move 2}

Swales vertritt die Ansicht, dass Move 2 folgende Funktionen erfüllt:

Most Move 2s establish a niche by indicating a gap-by showing that the research story so far is not yet complete. Move $2 \mathrm{~s}$ then are a particular kind of critique.

(Swales 2003: 186) 
Zudem hält Swales den Move 2 für einen notwendigen Textteil, der die Defizite der bisherigen Forschungen aufzeigt und darauf hinweist, was aus den vorliegenden Untersuchungen hervorgehen wird, um die entsprechenden Defizite zu füllen. Dazu Swales:

Move 2 is the key Move in Introductions. It is the hinge that connects Move 1 (what has been done) to Move 3 (what the present research is about). Move 2 thus establishes the motivation for the study. By the end of Move 2, the reader should have a good idea of what is going to come in Move 3.

(ebd.: 185-186)

\begin{tabular}{|l|l|l|}
\hline & Mit Move 2 & Ohne Move 2 \\
\hline DT & $96.67 \%(29)$ & $3.33 \%(1)$ \\
\hline CH & $53.33 \%(16)$ & $46.67 \%(14)$ \\
\hline Fisher's exact Test & $\mathrm{p}=0.0002$ & \\
\hline
\end{tabular}

Tabelle 5: Häufigkeiten vom Move 2 in deutschen und chinesischen Abschlussarbeiten

Aus Tabelle 5 wird ersichtlich, dass fast alle Abschlussarbeiten aus Deutschland und Österreich Move 2 behandelt haben (96,67\%), jedoch findet er sich nur in der Hälfte der Arbeiten aus Taiwan (53,33\%). Da fast alle deutschen Magisterarbeiten Move 2 behandeln, kann man festhalten, dass Move 2 als notwendiger Textteil der deutschsprachigen Magisterarbeiten angesehen wird. Wenn taiwanische Studierenden eine dem deutschen Verstehensprozess entsprechende Magisterarbeit schreiben wollen, sollten sie in der Magisterarbeit besonders darauf achten, Move 2 auszuführen.

Der Fisher's exact Test hat das obige Ergebnis als eindeutig bestätigt ( $\mathrm{p}=0,0002$, im Vergleich zum Standartwert $\mathrm{p}=0,001)$. Es besteht bei der Behandlung vom Move 2 ein hoch signifikanter Unterschied zwischen beiden Ländern.

Dieses Ergebnis stimmt mit der Behauptung von Kwan überein, dass in wissenschaftlichen Artikeln asiatischer Muttersprachler kaum Move 2 zu finden ist. Dazu Kwan:

Move 2 (Establishing a niche) has been found to be unnecessary in work that is published in less competitive research communities. The Counter-claiming step of Move 2 appears less regularly in articles that are circulated in some non-Anglophone or closely knit academic communities in which criticism of works by other members can be a threat to face.

(Kwan 2006: 32-33)

\begin{tabular}{|l|l|l|}
\hline & $\begin{array}{l}\text { Move 2 basiert auf } \\
\text { Erfahrungen }\end{array}$ & $\begin{array}{l}\text { Move 2 basiert auf der Analyse } \\
\text { der Fachliteratur }\end{array}$ \\
\hline DT & $13.33 \%(4)$ & $86.67 \%(26)$ \\
\hline CH & $70.00 \%(21)$ & $30.00 \%(9)$ \\
\hline Fisher's exact Test & $\mathrm{p}=0.0001$ & \\
\hline
\end{tabular}

Tabelle 6: Inhalt vom Move 2 in Magisterarbeiten beider Länder

Wird weiterhin darauf eingegangen, was die Studierenden beim Move 2 behandelt haben, ist zu erschließen, dass über die Hälfte der chinesischen Arbeiten die Defizite der Forschung auf der Basis persönlicher Erfahrungen darstellen (70,00\%). Dagegen basiert der Move 2 in deutschen Magisterarbeiten meistens auf der Analyse der Fachliteratur (86,67\%). Daran, dass der P-Wert $(p=0,0001)$ viel kleiner als der Standartwert $(p<0,001)$ beträgt, ersieht man, dass 
der Unterschied zwischen beiden Sprachen signifikant ist. Im folgenden Abschnitt werden diese Unterschiede anhand von Beispielen illustriert:

(Beleg 4) Move 2 basiert auf Erfahrungen:

Das folgende Beispiel stammt aus einer Magisterarbeit über das Wortschatzlernen der taiwanischen Deutschlerner. Dabei begründet die Verfasserin das Defizit der entsprechenden Forschung:

Die taiwanischen Deutschlerner haben oft Gedächtnisprobleme. Sie beschwerten sich oft, dass viele Fremdwörter zu lernen und diese schwer im Kopf zu behalten sind bzw. oft leicht vergessen werden. Aufgrund eigener Lernerfahrung und Beobachtungen der Verfasserin wird festgestellt, dass viele Lerner diese gleichen Probleme haben. Dieses kommt nicht nur beim Englisch-, sondern auch beim Deutschlernen vor. Deswegen ist es wichtig zu untersuchen, wie man auf einer schnellen, systematischen, einfachen und einer dem Gedächtnis günstige Weise eine Fremdsprache effektiver lernen kann. Dies ist ein wichtiges Thema [Übersetzung; C. S-L.].

(Chen 2007: 3)

An der Äußerung „Aufgrund eigener Lernerfahrung und Beobachtungen der Verfasserin wird festgestellt, dass viele Lerner diese gleichen Probleme haben“ erkennt man, dass das Forschungsdefizit aufgrund eigener Erfahrung und Beobachtung entwickelt worden ist.

Dagegen gehen die deutschsprachigen Magisterarbeiten meistens auf die Literatur ein (siehe Tabelle 6: 86,67\%) und ermitteln die Defizite der Forschungen zu Move 2 aus den Theorien. Swales (2003: 181) vertritt auch die Ansicht, dass eine wissenschaftliche Arbeit aufgrund der Einführung der Literatur auf die Defizite des Forschungsbereiches hinweisen soll, um die Stellung eigener Forschung zu etablieren:

Citations are used to create a research space for the citing author. By describing what has been done, citations point the way to what has not been done and so prepare a space for new research.

Der authentische deutschsprachige Beleg ist wie folgt:

(Beleg 5) Beispiel für den Move 2

In der vorliegenden Arbeit soll es vorwiegend um Probleme im morphologischen und im morphosyntaktischen Bereich gehen, da diese ein Kernproblem bei SLI darstellen (vgl. Guasti 2002). Dazu gehören unter anderem die Verbalflexion, Nominalflexion, Tempus, Kongruenz und Funktionswörter. Im Laufe der SLI Forschung sind viele Theorien über die zugrunde liegenden Ursachen dieser Sprachstörung entstanden, jedoch kann keine das volle Erscheinungsbild erklären.

(Wiesinger 2009: 9)

Daraus, dass fast bei jeder Aussage die Literatur als Beweis bzw. als theoretische Grundlage eingeführt wird, sieht man, dass im Gegensatz zu chinesischen, wo oft die Probleme bzw. die Fragestellung der Forschung aus eigener Erfahrung begründet werden, in deutschen Einleitungen jene eher aus der Analyse der Literatur abgeleitet werden. Ein weiteres Beispiel findet sich im deutschsprachigen Korpus bei Kollndorfer (2009: 5). 


\subsubsection{Move 1}

Sowohl deutsche als auch chinesische Abschlussarbeiten haben Move 1 behandelt. Jedoch legen sie Wert auf unterschiedliche Schwerpunkte:

\begin{tabular}{|l|l|l|}
\hline & Mit Step a vom Move 1 & Ohne Step a vom Move 1 \\
\hline DT & $26,67 \%(8)$ & $73,33 \%(22)$ \\
\hline CH & $93,33 \%(28)$ & $6,67 \%(2)$ \\
\hline Fisher's exact Test & $\mathrm{p}=0,0001$ & \multicolumn{2}{|l|}{} \\
\hline
\end{tabular}

Tabelle 7: Häufigkeiten von Step a des Move 1 in Magisterarbeiten beider Länder

Step a vom Move 1 behandelt "showing that the general research area is important, central, interesting, problematic, or relevant in some way" (Feak/Swales 2011: 55). Fast alle Arbeiten aus Taiwan behandeln den Step a vom Move 1 (93,33\%). Es ist ein signifikanter Unterschied zwischen den beiden Ländern festzustellen ( $\mathrm{p}=0,0001)$.

(Beleg 6) Beispiel für den Step a vom Move 1

In der folgenden chinesischen Magisterarbeit handelt es sich um die Bewertung digitaler Lehrwerke im Internet. Am Anfang der Einleitung stellt die Verfasserin dar, wie die Informationstechnik das Leben sowie das Lernen der Menschen beeinflusst, um die Wichtigkeit des behandelten Fachbereiches und -themas zu betonen:

In den letzten Jahren beeinflusst die Technik das Leben der Menschen, darunter ändert die Technik des Internets direkt das Leben, und auch die Erziehung der Menschen. Somit werden das Lehren und das Lernen in der traditionellen Weise auch beeinflusst ... Die Bücher sind nicht mehr die einzigen Möglichkeiten für den Erwerb von Kenntnissen [Übersetzung; C. S-L.].

(Su $2004: 1)$

Dieses Ergebnis stimmt mit dem Ergebnis der Tabelle 4 überein: Chinesische Einleitungen weisen oft mittels Darstellung der Motivation und des Zwecks (Tabelle 4: $22,92 \%+19,79 \%=42,71 \%$, vgl. Kapitel 4.1) auf die Wichtigkeit der jeweiligen Arbeit hin.

Dagegen behandeln nur 26,67\% der deutschen Abschlussarbeiten Step a. Stattdessen legen diese ihre Schwerpunkte meistens auf den Step b vom Move 1: ,introducing and reviewing items of previous research in the area“" (Feak/Swales 2011: 55).

(Beleg 7) Beispiel für den Step $b$ vom Move 1

Auf den ersten Blick stellt sich bei solch einer Arbeit zwangsläufig die Frage, was es zu dem Thema Metaphorik in der Sportberichterstattung noch Neues zu sagen gibt. Manche Autoren sind sogar der Meinung, dass es insgesamt schwer falle, einen Bereich innerhalb der Metaphorologie auszugrenzen, in dem man sinnvoll etwas Präzises (oder gar Neues) sagen zu können meint` (Strube 1991, 19). Auch die Untersuchung der Inhalte der Sportberichterstattung ist ein häufig bearbeitetes Forschungsgebiet (Loosen 1998, 8), doch diese Arbeit versucht im Gegensatz zu anderen Folgendes.

(Müller 2003: 1) ${ }^{8}$

\begin{tabular}{|l|l|l|}
\hline & Mit Step b vom Move 1 & Ohne Step b vom Move 1 \\
\hline DT & $90,00 \%(27)$ & $10,00 \%(3)$ \\
\hline
\end{tabular}

\footnotetext{
${ }^{8}$ Diese Magisterarbeit gibt die Literatur als Fußnote an. Um klar darzustellen, dass sie auf die Literatur hinweist, wird die Literatur hier direkt im Zitat angemerkt.
} 


\begin{tabular}{|l|l|l|}
\hline $\mathrm{CH}$ & $30,00 \%(9)$ & $70,00 \%(21)$ \\
\hline Fisher's exact Test & $\mathrm{p}=0,0001$ & \\
\hline
\end{tabular}

Tabelle 8: Vorkommen des Step b vom Move 1 in beiden Ländern

Tabelle 8 stellt dar, dass in Einleitungen chinesischer Magisterarbeiten aus Taiwan kaum Fachliteratur behandelt wird (70,00\%). Stattdessen fangen die deutschen Studierenden an, in den Einleitungen ihrer Magisterarbeiten Literatur zum Thema einzuführen. Dabei gehen sie darauf ein, inwiefern die bisherigen Forschungen das entsprechende Thema behandelt haben und welche Forschungsperspektive daraus entwickelt werden soll (90,00\%).

Dieses Ergebnis korreliert mit dem Ergebnis aus der Tabelle 6 (Move 2 das Aufzeigen der Forschungsdefizite aufgrund der Literatur in deutschen Magisterarbeiten: 86,67\%): Die deutschen Studierenden führen in ihren Einleitungen die Literatur über bisherige Forschungen des behandelten Themas ein und kommentieren deren Defizite. Aus dem Ergebnis der Tabelle 8 ersieht man, dass deutsche Einleitungen, im Gegensatz zu chinesischen, den Step b ("introducing and reviewing items of previous research in the area") signifikant häufiger behandeln $(\mathrm{p}=0,0001)$. Der Unterschied zwischen den Magisterarbeiten beider Länder ist sehr eindeutig.

Die deutschsprachigen Einleitungen behandeln ausführlicher die Diskussionen aus der Fachliteratur. Dieses Ergebnis geht eindeutig aus der Realisierung vom Move 1 Step $b$ ("introducing and reviewing items of previous research in the area", 90,00\%) und beim Move 2 (86,67\%) hervor. Dieses Resultat stimmt in einer indirekten Weise mit den Ergebnissen von Kaiser (2003) überein. Kaiser untersucht wissenschaftliche Texte von Studierenden aus Deutschland und aus Venezuela. Dabei hat sie entdeckt, dass die Seminararbeiten deutscher Studierenden die Literatur sowohl in weiterem Umfang als auch präziser behandeln als ihre KommilitonInnen aus Venezuela (2003: 321-322). Feak/Swales (2011: 55-56) halten den Step a vom Move 1 für fakultativ und den Step b für obligatorisch. Daraus lässt sich vermuten, dass deutsche Magisterarbeiten der Makrostruktur nach Swales 1990, 2004 und nach Feak/Swales 2011 (siehe Tabelle 1) vollständiger entsprechen als chinesische. Außerdem vertritt Swales (2003: 180) auch die Ansicht, dass in Einleitungen die Literatur der entsprechenden Forschungen behandelt werden soll:

Citations are used to show respect to previous scholars. They recognize the history of the field by acknowledging previous achievements.

Aus den obigen Ergebnissen (Tabellen 7 und 8) ist $\mathrm{zu}$ erschließen, dass chinesische Einleitungen beim Move 1 statt des Step $b$ fast nur den Step a behandelt haben. Da der Step $b$ gemäß der Makrostruktur nach Swales 1990, 2004 und nach Feak/Swales 2011 (siehe Tabelle 1) obligatorisch ist, sollen sie beim Move 1 statt des Step a eher den Step $b$ behandeln, um einer deutschen Magisterarbeit entsprechend zu schreiben.

\subsubsection{Move 3}

Nach Swales (2003) soll beim Move 3 darauf eingegangen werden, wie eigene Forschung dazu beiträgt, Defizite der bisherigen Forschung zu beseitigen. Dazu Swales (ebd.: 190):

The third and final step in the typical RP Introduction is to make an offer to fill the gap (or answer the question) that has been created in Move 2. 
(ebd.: 190)

\begin{tabular}{|l|l|l|}
\hline & Mit Step 1 vom Move 3 & Ohne Step 1 vom Move 3 \\
\hline DT & $10,00 \%(3)$ & $90 \%(27)$ \\
\hline CH & $66,67 \%(20)$ & $33,33 \%(10)$ \\
\hline Fisher's exact Test & $\mathrm{p}=0,0001$ & \\
\hline
\end{tabular}

Tabelle 9: Step 1 von Move 3 in beiden Sprachen

(Beleg 8) Beispiel für den Step 1 vom Move 3

Die folgende taiwanische Magisterarbeit behandelt das Thema „Blended Learning“ im DaFUnterricht und analysiert, wie man die Informationstechnik im traditionellen synchronen Unterricht einsetzt. Bei der Darstellung eigener Untersuchung (also beim Move 3) wiederholt die Verfasserin die Wichtigkeit der Arbeit:

Die neuen Medien werden immer häufiger beim Lehren eingesetzt. Deswegen versucht diese Arbeit die Umgebung des Lehrens, die Lehrstrategien, die Medien, die Elemente der Interaktionen zwischen dem Lehrer und den Lernern zu kombinieren, um einen deutschen „Blended Learning Kurs“ für taiwanische Hochschulen zu entwickeln. Diese Arbeit wird die traditionellen Lehrmethoden der Klassenzimmer mit dem E-Learning im Internet kombinieren, damit diese beiden eigene Lehrvorteile entfalten und somit den Lernern beim autonomen Lernen zu helfen [Übersetzung; C. S-L.].

(Yu 2008: 5)

Der Step 1 des Move 3 behandelt "announcing present research descriptively and/or purposively" (Swales 1990; Swales 2004; Feak/Swales 2011). Fast zwei Drittel der chinesischen Magisterarbeiten gehen mittels der Darstellung des Zwecks oder bei der Zielsetzung der Arbeit darauf ein, wie wichtig eigene Forschung für die wissenschaftliche Diskussion ist (66,67\%). Der Unterschied zwischen beiden Korpora ist eindeutig $(p=0,0001)$.

Dagegen behandeln deutsche Magisterarbeiten beim Move 3 kaum den Step 1. Stattdessen behandeln sie den Step 2 vom Move 3. Der Step 2 stellt, ,presenting research questions or hypotheses" (Swales 2004; Feak/Swales 2011) dar.

(Beleg 9) Beispiel für Hypothesen: Im folgenden Beleg listet die Magisterarbeit die Hypothesen auf:

Es werden vier Thesen aufgestellt, die zugleich den Handlungsrahmen für diese Arbeit bilden:

(1) Die Grenzen zwischen den Bereichen PR, Marketing und Werbung sind in der Praxis fließend.

(2) Pressemitteilungen entsprechen hinsichtlich ihres Aufbaus und ihrer Form der journalistischen Darstellungsform Nachricht.

(3) Die Pressemitteilung vereint die Informations- und die Persuasionsfunktion in sich.

(4) Die Pressemitteilungen über Produkte bzw. Dienstleistungen dienen primär dem werbenden Zweck.

(Müller 2011: 2)

Fragestellungen machen deutlich, welche Schwerpunkte die Magisterarbeiten behandeln wollen. Ein Beleg für die Behandlung der Fragestellungen sieht wie folgt aus:

(Beleg 10) Fragestellungen 
in Bezug auf den Körperkontakt in gleichgeschlechtlichen Freundschaften sind aber noch einige Fragen offen:

-Welche Arten von Berührungen gibt es in gleichgeschlechtlichen Freundschaften und welche

Körperteile sind involviert?

-Wie lässt sich Körperkontakt beschreiben?

-Welche Funktionen haben die unterschiedlichen Berührungen?

(Bernhardt 2007 : 1-2)

\begin{tabular}{|l|l|l|}
\hline & Mit Step 2 vom Move 3 & Ohne Step 2 vom Move 3: \\
\hline DT & $90,00 \%(27)$ & $10,00 \%(3)$ \\
\hline CH & $26,67 \%(8)$ & $73,33 \%(22)$ \\
\hline Fisher's exact Test & $\mathrm{p}=0,0001$ & \\
\hline
\end{tabular}

Tabelle 10: Häufigkeiten für Step 2 vom Move 3 in beiden Korpora

Die in Tabelle 10 dargestellten Ergebnisse zeigen, dass deutsche Magisterarbeiten beim Move 3 (also in den letzten Abschnitten der Einleitungen) fast immer den Step 2 (90,00\%), also die Fragestellungen und/oder die Hypothesen, behandeln. Stattdessen gehen die chinesischen nur in 26,67\% der Fälle auf den Step 2 ein. Dieses Ergebnis korreliert mit dem Ergebnis aus Tabelle 4. Dabei wurden in chinesischen Arbeiten weder Hypothesen (2,08\%) noch Fragstellungen (8,33\%) in nennenswerter Häufigkeit als selbständiges Unterkapitel in Einleitungen behandelt. Der Fisher's exact Test ratifiziert das obige Ergebnis als eindeutig $(\mathrm{p}=0,0001)$.

Der Step 2 vom Move 3 behandelt sowohl die Fragstellungen als auch die Hypothesen. Deswegen sollte man weiterhin der Frage nachgehen, wie häufig diese beiden vorkommen. $\mathrm{Da}$ in derselben Magisterarbeit Fragestellungen und Hypothesen gleichzeitig vorkommen können, werden im Folgenden diese beiden getrennt berechnet, um mehr darüber aussagen zu können. Zunächst wird auf die Hypothesen eingegangen:

\begin{tabular}{|l|l|l|}
\hline & Mit Hypothesen & Ohne Hypothesen \\
\hline DT & $40,00 \%(12)$ & $60,00 \%(18)$ \\
\hline CH & $6,67 \%(2)$ & $93,33 \%(28)$ \\
\hline Fisher's exact Test & $\mathrm{p}=0,0048$ & \\
\hline
\end{tabular}

Tabelle 11: Häufigkeit des Auftretens von Hypothesen in beiden Korpora

In den beiden Sprachen (DT: 40,00\%, CH: 6,67\%) werden Hypothesen in nur rund 45\% der Fälle formuliert. Dabei kommen die Hypothesen in deutschen Magisterarbeiten fast siebenfach häufiger als in chinesischen (DT 40,00\% vs. CH 6,67\%). Der Ratifizierungstest hat das obige Ergebnis als nicht überzufällig erwiesen. Der P-Wert des Fisher's exact Testes beträgt 0,0048 und ist größer als der Standartwert 0,001. Der Unterschied zwischen den beiden ist somit auf diesem Niveau nicht signifikant. Der Grund könnte darin liegen, dass in deutschen wie chinesische Magisterarbeiten mehrheitlich keine Hypothesen behandeln (DT $60,00 \%$ vs. $\mathrm{CH} \mathrm{93,33 \% ).}$

Um die Hypothesen für die Analyse zu erstellen, muss man die Ergebnisse voraussagen können und mittels der Hypothesen die Richtigkeit der Ergebnisse überprüfen. Deswegen ist es nicht einfach, die Hypothesen zu erstellen. Aus diesem Grund kommen sie im Vergleich zu Fragestellungen seltener in Einleitungen der Magisterarbeiten vor. 


\begin{tabular}{|l|l|l|}
\hline DT & $80,00 \%(24)$ & $20,00 \%(6)$ \\
\hline CH & $26,67 \%(8)$ & $73,33 \%(22)$ \\
\hline Fisher's exact Test & $\mathrm{P}=0,0001$ & \\
\hline
\end{tabular}

Tabelle 12: Häufigkeit der formulierten Fragestellungen in beiden Korpora

In deutschen Magisterarbeiten kommen explizit formulierte Fragestellungen fast dreifach häufiger als in chinesischsprachigen (DT: $80,00 \%$ vs. $\mathrm{CH}: 26,67 \%$ ) vor. Betrachtet man die in Tabelle 11 und in Tabelle 12 genannten Ergebnisse zusammen, wird ersichtlich, dass Hypothesen (Tabelle 11: 40,00\%) plus Fragestellungen (80,00\%) in deutschsprachigen Magisterarbeiten viel häufiger vorkommen als in chinesischen (Tabelle 11: 6,67\% und Tabelle 12: 26,67\%). An der hohen Anzahl der explizit formulierten Fragestellungen $(80,00 \%)$ erkennt man, dass die Fragestellung als notwendiger Textteil deutscher Abschlussarbeiten gesehen wird. Der Fisher's exact Test stützt dieses Ergebnis sehr eindeutig ( $p=0,0001)$.

Daran, dass 96,67\% der deutschen Magisterarbeiten den Move 2 (vgl. Tabelle 5 über Defizite der bisherigen Forschung) und 90,00\% der deutschen den Step 2 des Move 3 (vgl. Tabelle 10 über die Fragestellungen und/ oder Hypothesen) behandeln, wird ebenfalls deutlich, dass beide als notwendige Teile deutscher Einleitungen gesehen werden.

\begin{tabular}{|l|l|l|}
\hline & Mit Step 7 vom Move 3 & Ohne Step 7 vom Move 3 \\
\hline DT & $100 \%(30)$ & $0 \%(0)$ \\
\hline CH & $46,67 \%(14)$ & $53,33 \%(16)$ \\
\hline Fisher's exact Test & $\mathrm{p}=0,0001$ & \\
\hline
\end{tabular}

Tabelle 13: Step 7 vom Move 3 in beiden Sprachen

Der Step 7 des Move 3 behandelt "outlining the structure of the paper" (Swales 1990, 2004, 2011). Nach Swales (2003) gehört die „Aufbaustruktur der Arbeit“ für die englische Magisterarbeit nicht zum obligatorischen Textteil. Aber sie ist für eine englische Dissertation und in Fachbereichen wie Informatik wichtig. Dazu Swales:

A final option is to consider whether you need to explain how your text is organized. This element is obligatory in dissertations, but is only included in RPs under certain circumstances... Cooper (1985) found, for example, that outlining the RP structure was quite common in computer technology.

(ebd.: 194)

(Beleg 11) Aufbau der Arbeit

Die vorliegende Arbeit sucht grammatikalisch flexibles Verhalten von SprecherInnen zu beschreiben, sein Zustandekommen zu erklären sowie Möglichkeiten der Analyse aufzuzeigen. $\mathrm{Zu}$ diesem Zweck soll zunächst in einem theoretischen Teil (Kapitel 2) ein vorbereitendes Fundament gelegt werden... Kapitel 2.1 wird sich dabei mit grundsätzlichen Unterschieden zwischen gesprochener und geschriebener Sprache sowie mit der Frage, ob beide Medien auf demselben Sprachsystem beruhen.

(Diewald 2010: 4)

Am Ende der Einleitungen gehen fast alle deutschen Abschlussarbeiten auf die Aufbaustruktur der Darstellung ein (100\%), während weniger als die Hälfte der chinesischen Magisterarbeiten diese darstellen (46,67\%). Daran erkennt man, dass der Aufbau ein notwendiger Textteil deutschsprachiger Abschlussarbeiten ist. Dieser Unterschied zwischen den beiden Ländern ist signifikant ( $\mathrm{p}=0,0001)$. 


\section{$5 \quad$ Fazit und Ausblick}

Dieser Beitrag vergleicht chinesische mit deutschen Abschlussarbeiten, um deren Unterschiede bezüglich der Makrostrukturen und der inhaltlichen Schwerpunkte zu ermitteln. Dabei ergänzen sich die qualitative Move Analysis nach Swales und die quantitative Analyse. Sowohl deutschsprachige als auch chinesischsprachige Einleitungen entsprechen der Makrostruktur nach Swales $(1990,2004)$ und nach Feak/Swales (2011, siehe Tabelle 1) und behandeln alle drei Moves. Jedoch behandeln sie die einzelnen Steps der Moves mit unterschiedlicher Häufigkeit:

Beim Move 1 behandeln die meisten chinesischsprachigen Magisterarbeiten den Step a, um die Wichtigkeit des Untersuchungsbereiches darzulegen, während die deutschen Studierenden in Einleitungen besonderen Wert auf den Step $b$ legen, d. h. auf die Fachliteratur des Forschungsthemas eingehen.

Beim Aufzeigen der Forschungsdefizite (Move 2) besteht der Unterschied zwischen beiden Korpora darin, dass im Gegensatz zu deutschsprachigen Abschlussarbeiten (vgl. Tabelle 5: 96,67\%) nur die Hälfte der chinesischsprachigen Einleitungen Move 2 behandeln (vgl. Tabelle 5: 53,33\%). Die deutschsprachigen Arbeiten kommentieren die Defizite meistens aufgrund der behandelten Literatur und im Gegensatz dazu die chinesischsprachigen oft aufgrund eigener Beobachtungen oder Erfahrungen.

Beim Move 3 gehen chinesischsprachige Arbeiten besonders auf den Step 1, also eigene Untersuchung gezielt anzukündigen (vgl. Tabelle 9), ein, während deutschsprachige Arbeiten meistens den Step 2 behandeln (Hypothesen/Fragestellungen, vgl. Tabelle 10). Daran, dass chinesischsprachige Arbeiten sowohl beim Move 1 den Step a (Wichtigkeit des Forschungsbereiches) als auch bei Move 3 den Step 1 (Ankündigung eigener Untersuchungszwecks und somit das Aufzeigen der Wichtigkeit/Notwendigkeit eigener Forschung) behandeln, wird klar, dass sie viel Wert auf die Betonung der Wichtigkeit/Notwendigkeit der eigenen Forschung legen.

Wenn man eine dem deutschen Verstehensprozess entsprechende Abschlussarbeit schreiben will, muss man in der Einleitung die drei von Swales $(1981,1990,2004)$ genannten Moves behandeln. Beim Move 1 soll man den Step $b$ behandeln, indem man die Literatur über den Untersuchungsbereich oder über das Forschungsthema begrifflich kurz darstellt und analysiert. Aufgrund dieser Analyse der Literatur weist man auf die Defizite bzw. auf die Kommentare in der bisherigen Diskussion (Move 2) hin. Beim Move 3, wo die eigene Forschung analysiert wird, sollte man darauf eingehen, wie man versucht, die oben genannten Forschungslücken zu beseitigen. Dabei stellen deutsche Magisterarbeiten meistens entweder die Fragestellungen und/oder die Hypothesen eigener Forschung dar (Move 3, Step 2). Am Ende der Einleitungen wird auf die Aufbaustruktur der Arbeit eingegangen.

Dieser Artikel untersucht nur wissenschaftliche Arbeiten von Studierenden. Wenn man in zukünftigen Untersuchungen dazu noch die Ansichten der Betreuer und die der VerfasserInnen der Abschlussarbeiten analysiert, werden die unterschiedlichen Forschungsperspektiven und Forschungskonzeptionen deutlich, die vermutlich hinter den textuellen Entscheidungen der Studierenden stehen. 


\section{Literaturverzeichnis}

Biber, Douglas/Connor, Ulla/Upton, Thomas A. (2007): Discourse on the Move: Using Corpus Analysis to Describe Discourse Structure. Amsterdam: Benjamins.

Bharadwaj, Anandhi (2000): "A resource-based perspective on Information technology capability and firm performance: An empirical investigation”. MIS Quarterly 24/1: 169196.

Clyne, Michael (1987): "Cultural Differences in the Organization of Academic Texts: English and German". Journal of Pragmatics 11: 211-247.

Dittmann, Jürgen et al. (2003): „Schreibprobleme im Studium - Eine empirische Untersuchung“. In: Ehlich, Konrad/Steets, Angelika (eds.): Wissenschaftlich schreiben lehren und lernen. Berlin, de Gruyter: 51-65.

Ehlich, Konrad/Steets, Agelika (eds.) (2003): Wissenschaftlich schreiben -lehren und lernen. Berlin: de Gruyter.

Feak, Christine/Swales, John Malcolm (2011): Creating Contexts: Writing Introductions across Genres. Ann Arbor: University of Michigan Press.

Fischer, Almut/Moll, Melanie (2002): „Die Seminararbeit als Einstieg ins wissenschaftliche Schreiben“. In: Redder, Angelika (ed.): Effektiv studieren. Texte und Diskurse an der Universität. Duisburg, Gilles/Francke: 135-165.

Fisher, Ronald Aylmer (1992): "On the interrelation of $\chi 2$ from contingency tables, and the calculation of P." Journal of the Royal Statistical Society 85: 87-94.

Graefen, Gabriele (1997): Der Wissenschaftliche Artikel - Textart und Textorganisation. Frankfurt a. M.: Lang.

Gruber, Helmut (2010): „Modelle des wissenschaftlichen Schreibens: Ein Überblick über zentrale Ansätze und Theorien“. In: Saxalber, Annemarie/Esterl, Ursula (eds.): Schreibprozesse begleiten. Vom schulischen zum universitären Schreiben. Innsbruck, Studien: $17-40$.

Gruber, Helmut/Birgit Huemer (2008): "Two views on text structure: Using rhetorical structure theory and register und genre theory in improving students' academic writing". Odense Working Papers in Language and Communication 29: 332-365.

Gruber, Helmut et al. (2006): Genre, Habitus und wissenschaftliches Schreiben. Eine empirische Untersuchung studentischer Texte. Wien: LIT.

Hsiao, Cheng-Hua/Yu, Hsueh-Ying (2012): "Knowledge presentation in thesis writing Examining Move use in reviewing literature". Teaching English and Learning 36/3: 133179.

Huemer, Birgit (2014): „Der wissenschaftliche Artikel in der Disziplin germanistische Linguistik“. In: Wissenschaftliches Schreiben im Deutschen und im Chinesischen. Sammelband zum Österreich-taiwanischen Symposium 2014: 117-130.

Hussy, Walter/Schreier, Margrit/Echterhoff, Gerald (2010): Forschungsmethoden in Psychologie und Sozialwissenschaften. Berlin/Heidelberg: Springer.

Kaiser, Dorothee (2003): „'Nachprüfbarkeit' versus 'Originalität' - Fremdes und Eigenes in studentischen Texten aus Venezuela und Deutschland“. In: Ehlich, Konrad/Steets, Agelika/Traunspurger, Irene (eds.): Wissenschaftlich schreiben - lehren und lernen. Berlin, de Gruyter: 305-324. 
Koehn, Philipp (2000): “German-English Parallel Corpus 'de-news"”. Daily News 1996-2000. http://homepages.inf.ed.ac.uk/pkoehn/publications/de-news/ [10.12.2013].

Kruse, Otto/Jakobs, Eva-Maria (1999): „Schreiben lernen an der Hochschule: Ein Überblick“. In: Kruse, Otto/Jakobs, Eva-Maria/Ruhmann, Gabriela (eds.): Schlüsselkompetenz Schreiben. Konzepte. Methoden. Projekte für Schreibberatung und Schreibdidaktik an der Hochschule. Neuwied Kriftel, Luchterhand: 19-34.

Kwan, Becky Siu Chu (2006): "The schematic structure of literature reviews in doctoral theses of applied linguistics". English for Specific Purposes 25: 30-55.

Li, Huiyan (2010): „Kontrastiver Vergleich zwischen den englischen und den chinesischen Einleitungen in sozialwissenschaftlichen Fachartikeln“. In: Journal of Chongqing University of Technology 24/11: 110-114.

Loi, Chek Kim/Evans, Moyra Sweetnam (2010): "Cultural differences in the organization of research article introductions from the field of educational psychology: English and Chinese". Journal of Pragmatics 42: 2814-2825.

Moll, Melanie (2002): „'Protokollieren' statt 'stenografieren' - Das Protokoll als zusammenfassende Verschriftlichung einer Seminarversantaltung". In: Redder, Angelika (ed.): Effektiv studieren. Texte und Diskurse an der Universität. Duisburg, Gilles/Francke: 85-103.

Moore, David (2002): Statistic: Concepts and Controversies. New York: Freeman.

Pohl, Thorsten (2007): „Studien zur Ontogenese wissenschaftlichen Schreibens“. Germanistische Linguistik 271. Tübingen, Niemeyer: xii, 621.

Redder, Angelika (2002): Effektiv studieren. Texte und Diskurse an der Universität. Duisburg: Gilles/Francke.

Sachtleber, Susanne (1990): „Linearität vs. Digressivität. Wissenschaftliche Texte im zweisprachigen Vergleich“. Folia Linguistica 24: 105-122.

Şenöz-Ayata, Canan (2012): „Interkulturelle Wissenschaftskommunikation - dargestellt an Abstracts in deutschen und türkischen Germanistikzeitschriften“. Linguistik online 52/2: $31-57$.

Steinhoff, Torsten (2007): Wissenschaftliche Textkompetenz. Sprachgebrauch und Schreibentwicklung in wissenschaftlichen Texten von Studenten und Experten. Tübingen: Niemeyer.

Swales, John Malcolm (1981): Aspects of article introductions. Birmingham: LSU, University of Aston.

Swales, John Malcolm (1990): Genre analysis: English in academic and research settings. Cambridge : Cambridge University Press.

Swales, John Malcolm (2003): Academic writing for graduate students: essential tasks and skills: a course for nonnative speakers of English. Ann Arbor: University of Michigan Press.

Swales, John Malcolm (2004): Research Genres: Explorations and Applications. Cambridge: Cambridge University Press.

Swales, John Malcolm (2011): Aspects of Article Introduction. Michigan Classics Edition. Ann Arbor: The University of Michigan Press.

Szurawitzki, Michael (2013): „Evaluierende Conclusions - Untersuchung germanistischer studentischer Hausarbeiten zur linguistischen Wissenschaftssprache“. In: Tinnefeld, 
Thomas (ed.): Hochschulischer Fremdsprachenunterricht. Saarbrücker Schrift zur Linguistik und Fremdsprachendidaktik. Htw saar, Saarbrücken: 347-358.

Thielmann, Winfried (2009): Deutsche und englische Wissenschaftssprache im Vergleich. Hinführen - Verknüpfen - Benennen. Heidelberg: Synchron.

Tzilinis, Anastasia (2013): Sprachliches Handeln im deutschen wissenschaftlichen Artikel. Ein Beitrag zur Komparatistik der Wissenschaftssprachen. Krottenmühl: Synchron.

Xiao, Richard (2008): "Well-known and influential corpora. In: Lüdeling, Anke/Kytö, Merja (eds.): Corpus linguistics: An international handbook 1. Berlin, de Gruyter: 383-457.

Zaleska, Maria (2010): „Schluss ohne Schlussfolgerungen? Schlussabschnitte sprachwissenschaftlicher Artikel im Vergleich“. In: Heller, Dorothee (ed.): Deutsch, Italienisch und andere Wissenschaftssprachen. Schnittstellen ihrer Analyse. Frankfurt a. M., Lang: 151-179.

\section{Korpus}

Bernhardt, Maike ( 2007): Berührung als Kommunikation. Eine linguistische Untersuchung zu Form und Funktion von Berührungen in gleichgeschlechtlichen Freundschaften. Magisterarbeit, Universität Hamburg.

Chen, Liang-Hsu (2012): Interkulturelles Lernen taiwanischer Studierender am Beispiel eines Lektürekurses mit ausgewählten Märchen der Gebrüder Grimm. Magisterarbeit, Fu-Jen Uni. Taipei, Taiwan R.O.C.

Chen, Yen-Chun (2007): Eine Untersuchung zur Anwendung von Gedächtnisstrategien beim Lernen des deutschen Wortschatzes. Magisterarbeit, National Kaohsiung First University of Science and Technology, Kaohsiung, Taiwan R.O.C.

Chin, Yu-Shu (2010): The methods of teaching word formation in German as Foreign Language: Prefix verbs exercises in textbooks „Themen aktuell" and „Lagune“. Magisterarbeit, Chinese Culture Uni. Taipei/Taiwan.

Diewald, Katharina (2010): Grammatikalische Flexibilität in der verbalen Interaktion. Am Beispiel von Apokoinukonstruktionen. Diplomarbeit zum Magister der Philosophie, Universität Wien.

Faulhaber, Jaroslav (2013): Mehrsprachigkeit und die Bedeutung von Heritage Language Education an österreichischen Universitäten. Diplomarbeit zum Magister der Philosophie, Universität Wien.

Huang, Ching-Shih (2003): The Roll of Culture in the Textbooks of German Language Lessons. Magisterarbeit, National Kaohsiung First University of Science and Technology, Kaohsiung, Taiwan R.O.C.

Huang, Sheng-Fang (2004): German-Chinese Translation in Instruction Texts - Quality Management Manual as Example. Magisterarabeit, Fu-Jen University, Taipei/Taiwan.

Kollndorfer, Kathrin (2009): Entwicklung der Modalität im Erstspracherwerb des Deutschen. Diplomarbeit zum Magister der Philosophie, Universität Wien.

Lin, I-Yen (2005): Zur Untersuchung der Übersetzungsprobleme aus der Perspektive interkultureller Kommunikation - Am Beispiel des Buches „Europäische Esskultur“ von Günther Hirschfelder. Magistgerarbeit, National Kaohsiung First University of Science and Technology, Kaohsiung, Taiwan R.O.C. 
Müller, Franziska (2011): Die Pressemitteilung - pure Werbung? Eine textlinguistische Analyse zu Interdependenzbeziehungen zwischen Public Relations und Werbung. Magisterarbeit, Universität Leipzig.

Müller, Wolfgang (2003): Vom „Länderkampf“ zum „Länderspiel“. Zur Metaphorik in der Sportberichterstattung. Eine Analyse von Texten in deutschen Tageszeitungen von 1934 bis 2002. Magisterarbeit, Universität Hamburg.

$\mathrm{Su}$, Chiu-Hua (2004): Development of German Web-based Courseware for German Beginners. Magisterarbeit, National Kaohsiung First University of Science and Technology, Kaohsiung, Taiwan R.O.C.

Tsai, Wen-Shu (2009): Kritik zur chinesischen Übersetzung von Siegfried Lenz Erzählung ,, So zärtlich war Suleyken", basierend auf der Theorie von Katharina Reiss. Magisterarbeit, National Kaohsiung First University of Science and Technology, Kaohsiung, Taiwan, R.O.C

Wiesinger, Julia (2009): Morphologische und Morphosyntaktische Auffälligkeiten bei Spezifischer Sprachentwicklungsstörung in verschiedenen Sprachen. Diplomarbeit zum Magister der Philosophie, Universität Wien.

Yu, Ching-Ping (2008): Blended Learning im DaF-Unterricht Möglichkeiten und Schwierigkeiten - Ein Beispiel im Audio-Visuellen Unterricht. Magisterarbeit, National Kaohsiung First University of Science and Technology, Kaohsiung, Taiwan R.O.C. 